\title{
The High Energy Region of the Absorption Edge of a-Si:H, a Theoretical Study-III
}

\author{
Salwan Kamal Jamil Al-Ani" ${ }^{*}$, Abdullah Ibrahim, Subhi Saeed Al-Rawi
}

Physics Department, College of Science for Women, Baghdad University, Baghdad, Iraq.

Email: *salwan_kamal@yahoo.com

Received May $4^{\text {th }}, 2012$; revised June $8^{\text {th }}, 2012$; accepted July $12^{\text {th }}, 2012$

\begin{abstract}
In this paper we try to give a reasonable account for the origin of the experimental optical energy gap $E_{o}$ of a-Si:H deduced from the plot due to Cody ( $\varepsilon_{2}^{1 / 2}$ vs. $E$ ). Using a realistic model density of states diagram for a-Si:H and the constant dipole matrix element assumption, and a reasonable definition of the real optical energy gap $E_{G}$, a new theoretical equation for $\varepsilon_{2}(E)$ was derived. The plot of the square root of this function $\varepsilon_{2}^{1 / 2}$ as a function of the photon energy $E$ for appropriate fitting parameters gives a straight line fit in the energy region of significance extrapolating to the energy axis at a value similar to the experimental optical gap but about $0.1 \mathrm{eV}$ lower than the theoretical optical gap $E_{G}$ proposed in our paper. We conclude that the experimental optical gap $E_{o}$ does not necessarily coincide with any optical transition threshold in the density of states diagram of a-Si:H.
\end{abstract}

Keywords: High Energy; Absorption Edge

\section{Introduction}

In two previous papers [1,2], we concluded from the re-analysis of the experimental results of Jackson et al. [3] for the density of states convolution integral $J(E)$ as a function of photon energy $E$ for GD a-Si:H in the energy range $(1.6-3.7 \mathrm{eV})$, that the theoretical model due to Cody [4] is the suitable theoretical model for the interpretation of the optical data at the high absorption region of the optical absorption edge of this important material.

This model assumes a parabolic density of states (DOS) distribution near each of the valence and conduction band edges (similar to the Tauc [5] model), and a constant dipole matrix element (Tauc assumed a constant momentum matrix element).

The problem of the interpretation of the optical energy gap $E_{\text {opt }}$ is still a matter of controversy in literature $[4,6]$. For example in the case of our analysis, the optical gap obtained from the plot attributed to Cody $\left(\varepsilon_{2}^{1 / 2}\right.$ vs. E) which is $\sim 1.68 \mathrm{eV}$ does not match with the value of the mobility gap of Jackson et al. [3] samples i.e. $\sim 1.93 \mathrm{eV}$. While the gap obtained from the famous Tauc plot $\left(E \varepsilon_{2}^{1 / 2}\right.$ vs. $E$ ) $\sim 1.89 \mathrm{eV}$ is significantly closer to the value of the mobility gap for Jackson et al. samples.

In this paper we try to give a reasonable explanation for this problem, which we hope that it gives a possible

\footnotetext{
"Corresponding author.

"Present address: NCPW, P.O. Box (25777), Doha, Qatar.
}

clue towards the understanding of the problem of the interpretation of the optical energy gap problem in amorphous semiconductors.

\section{Theory}

The imaginary part of the dielectric constant $\varepsilon_{2}(E)$ for amorphous semiconductors is given by [3]:

$$
\varepsilon_{2}(E)=\text { const } R^{2}(E) J(E)
$$

where $R^{2}(E)$ is the normalized average dipole matrix element and $J(E)$ is defined as:

$$
J(E)=\int N_{V}(E) N_{C}\left(E^{\prime}+E\right) \mathrm{d} E
$$

where $N_{v}\left(E^{\prime}\right)$ and $N_{c}\left(E^{\prime}\right)$ are the valence and conduction band density of states functions respectively and $E^{\prime}$ is the state energy.

It usually assumed that the density of states distribution near each of the valence and conduction band edges is some simple power law i.e. $N\left(E^{\prime}\right) \alpha E^{m}$. If $R^{2}(E)$ also obeys a simple power law of the form $R^{2}(E) \alpha E^{-q}$. The general solution of Equation (1) using the above assumptions is [4]:

$$
\varepsilon_{2}(E)=K E^{-q}\left(E-E_{o}\right)^{r}
$$

where $K$ is a constant, $r=2 m+1$ ( for symmetrical DOS), and $E_{o}$ is a parameter usually identified with the optical energy gap $E_{\text {opt }}\left(E_{o}=E_{\text {opt }}\right)$ though of course this is not 
necessarily true.

For the Tauc model [5] $N\left(E^{\prime}\right) \alpha E^{\prime 1 / 2}$ and the momentum matrix element squared $\left(P^{2}(E)\right)$ is constant, thus $q=2$ and $r=2$ in Equation (3), then the relation $E \varepsilon_{2}^{1 / 2}$ $=A\left(E-E_{o}\right)$ gives a straight line with $E_{o}$ as the Tauc gap.

For the Cody approach [4] the only variant with the Tauc approach is the assumption of a constant dipole matrix element $R^{2}(E)=$ const., Thus $q=0$ and $r=2$ in Equation (3), then the relation $\varepsilon_{2}^{1 / 2}=B\left(E-E_{o}\right)$ gives a straight line with $E_{o}$ different from the Tauc gap usually lower.

The optical energy gap $E_{o}$ is obtained from the extrapolation of the straight line to the photon energy axis.

In this paper our main concern is with interpretation of $E_{o}$ using a detailed model density of states for a-Si:H assuming a reasonable theoretical optical energy gap in order to deduce a new equation for $\varepsilon_{2}(E)$.

\section{Our Theoretical Analysis}

Figure 1 depicts the model density of states diagram based mainly on the standard model applied for electronic quality a-Si:H. Here we take every possible detail into account accept for the effect of exponential tails on the resulting $E_{o}$ which was studied previously by Malik and O'Leary [7]. In this figure we note the following:

1) The density of states distribution in the extended states near each of the valence and conduction band edges $E_{v}$ and $E_{c}$ respectively is parabolic $\left(N\left(E^{\prime}\right) \alpha E^{\prime 1 / 2}\right)$.

2) In the localized states regions just under the conduction band edge $\left(E_{c}-E_{c}^{\prime}=\Delta E^{\prime \prime}\right)$, and just above the valence band edge $\left(E_{v}^{\prime}-E_{v}=\Delta E^{\prime \prime}\right)$ the density of states distributions are also parabolic.

3) In the regions far away from each of the band edges, the density of states distributions is exponential in nature.

4) The energy interval $(\Delta E)$ in Figure 1 is $\left(E_{c}-E_{A}=\right.$ $\left.E_{B}-E_{v}\right)$ i.e. the density of states diagram is assumed to be symmetric.

5) The energy interval $\left(\Delta E^{\prime}\right)$ in the same figure is $\left(E_{c}^{\prime}\right.$ $\left.-E_{A}=E_{B}-E_{v}^{\prime}\right)$.

6) The real optical energy gap which is defined as $E_{G}$ represents in this diagram the assumed threshold for optical transitions responsible for the high energy region of the absorption edge which is $\left(E_{c}^{\prime}-E_{v}\right.$ or $\left.E_{c}-E_{v}^{\prime}\right)$.

This last definition of $E_{G}$ is based on the experimental findings of Jackson et al. [3] that the matrix element is nearly the same for localized-extended and extendedextended transitions. We call $E_{G}$ the real optical gap because it is defined from the density of states diagram and not from the analysis of optical data i.e. $E_{\text {opt }}$, there is no a priori necessity or assuming them equal.

According to Figure 1:

$$
\begin{aligned}
& N_{V}\left(E^{\prime}\right)=N\left(E_{V}^{\prime}\right)\left\{E_{B}-E^{\prime} / \Delta E_{V}\right\}^{1 / 2} \\
& N_{C}\left(E^{\prime}\right)=N\left(E_{C}^{\prime}\right)\left\{E^{\prime}-E_{A} / \Delta E_{C}\right\}^{1 / 2}
\end{aligned}
$$

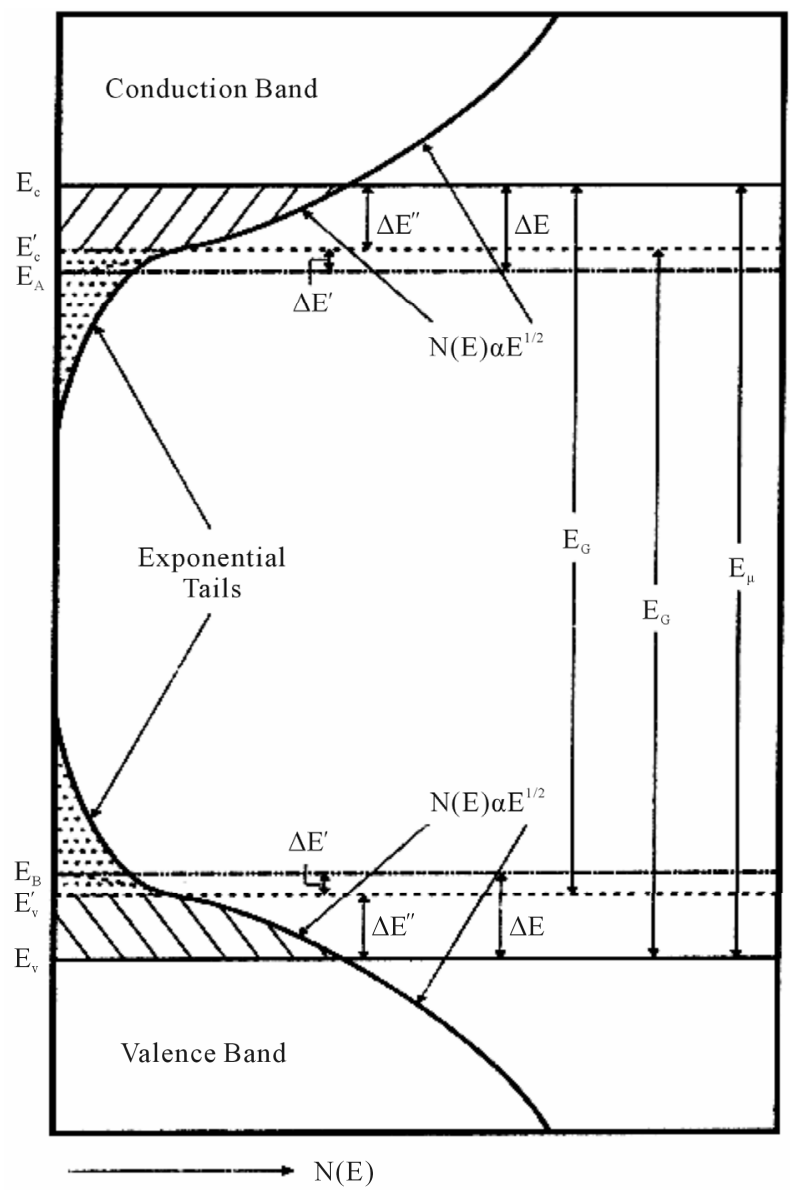

Figure 1. The model density of states diagram of a-Si:H.

where $N\left(E_{v}^{\prime}\right)$ and $N\left(E_{c}^{\prime}\right)$ are the densities of states at the valence and conduction band edges respectively.

For amorphous silicon and un-polarized light the prefactor in Equation (1) is equal to $(2 \pi e)^{2} R^{2}(E) / 3 \rho_{A}$ [3] where $\rho_{A}$ is the atomic density, taking this into account we substitute Equations (2) and (4) in Equation (1) to get [6]:

$$
\varepsilon_{2}(E)=\frac{(2 \pi e)^{2} R^{2}(E) N^{2}\left(E_{C}^{\prime}\right)}{3 \rho_{A} \Delta E}\left(E-E_{G}\right)^{2} \cdot f(E)
$$

where:

$$
\begin{aligned}
f(E)= & \left(E-E_{G}\right)^{2}\left[\frac{b(E)-2}{4} \sqrt{b(E)+c(E)-1}\right. \\
& \left.-\frac{b(E)}{4} \sqrt{c(E)}-\left(\frac{b^{2}(E)+4 c(E)}{8}\right)\right] \\
& \sin ^{-1}\left[\frac{2-b(E)}{\sqrt{b^{2}(E)+4 c(E)}}\right] \\
+ & \sin ^{-1}\left[\frac{b(E)}{\sqrt{b^{2}(E)}+4 c(E)}\right] \cdot(6-a)
\end{aligned}
$$




$$
\begin{gathered}
b(E)=1-\frac{\Delta E-\Delta E^{\prime}}{E-E_{G}} \\
c(E)=\frac{\Delta E}{E-E_{G}}\left[1+\frac{\Delta E^{\prime}}{E-E_{G}}\right]
\end{gathered}
$$

We see that Equation (5) includes three main parameters of the model density of states of Figure $1 E_{G}, \Delta E$ and $\Delta E^{\prime}, \Delta E-\Delta E^{\prime}$ in Equation (6b) is just $\Delta E^{\prime \prime}$.

For a-Si considering $\rho_{A}$ is that for c-Si, Equation (5) becomes:

$$
\varepsilon_{2}(E)=0.43 \times 10^{-44} R^{2}(E) \frac{N^{2}\left(E_{C}\right)}{\Delta E} f(E)
$$

where $R^{2}(E)$ is in units of $\AA^{2}, E$ in $\mathrm{eV}$, and $N\left(E_{c}\right)$ is in units of $\mathrm{eV}^{-1} \cdot \mathrm{cm}^{-3}$

\section{Results and Discussion}

The function $f(E)$ is plotted for chosen parameters ( $X=$ $\left.E_{G}\right)=1.78 \mathrm{eV},(Y=\Delta E)=0.18 \mathrm{eV}$ and $\left(Z=\Delta E^{\prime}\right)=0.03$ $\mathrm{eV}$ and the result is depicted in Figure 2.

If we plot the square root of this function i.e. $\sqrt{f(E)}$ as function of $E$, then Figure 3 gives a straight line that fits the equation $0.654 \mathrm{E}-1.68$, we see that the extrapolation to the $\mathrm{x}$-axis is equal to $\sim 1.68 \mathrm{eV}$ which is equal to that for Jackson et al. [3] samples.

Thus although the assumed real optical gap $E_{G}$ in our model was $1.78 \mathrm{eV}$ the optical gap that results from the $(f(E))^{1 / 2}$ vs. $E$ ) plot is $1.68 \mathrm{eV}$ which $0.1 \mathrm{eV}$ smaller. Thus we conclude that the experimental optical energy gap $E_{\text {opt }}$ is not an accurate marker of the energy gap responsible for the threshold of optical transitions responsible for the high energy region of the absorption edge.

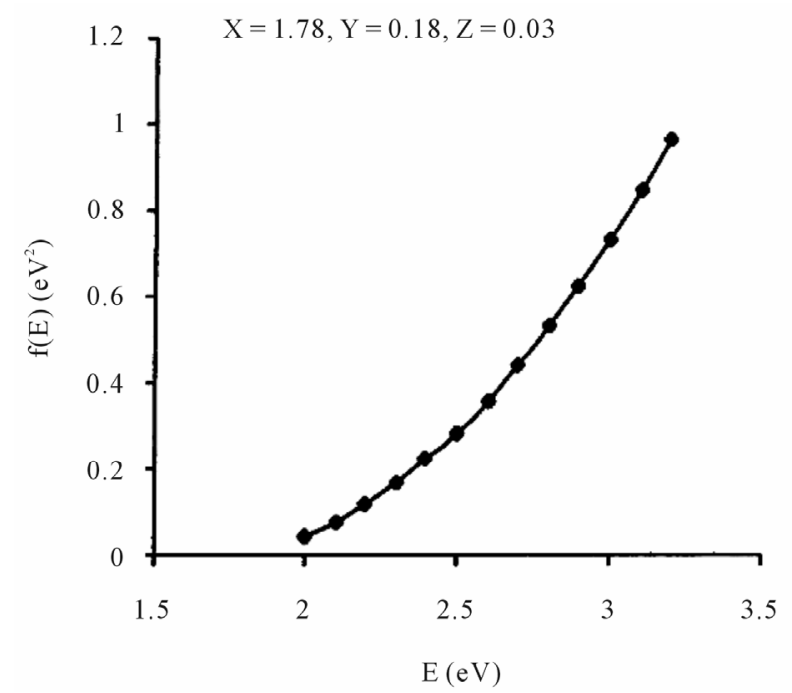

Figure 2. Plot of the function $f(E)$ vs. $E$ for the fitting paramers $X=E_{G}=1.78 \mathrm{eV}, Y=\Delta E=0.18 \mathrm{eV}, Z=\Delta E^{\prime}=0.03$ eV.

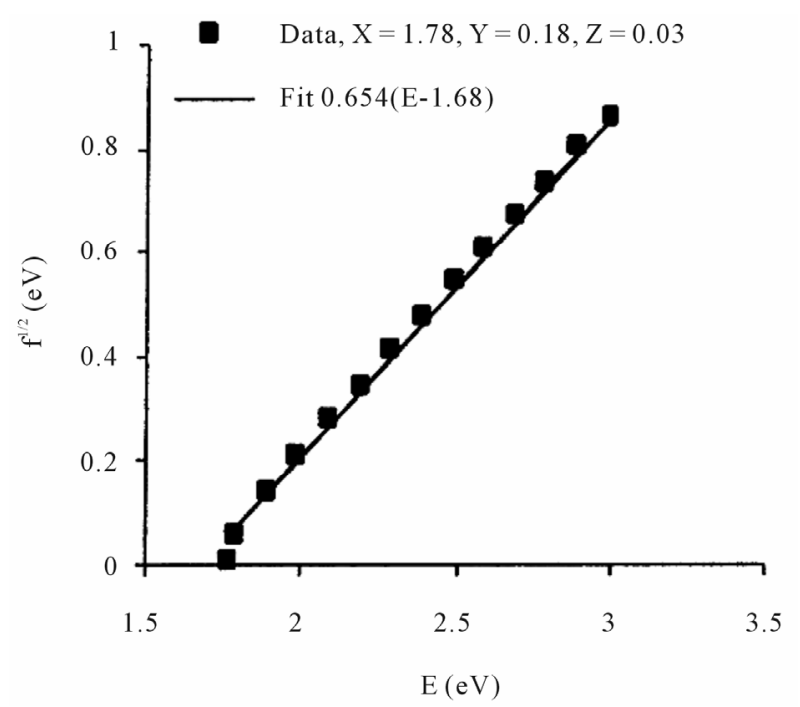

Figure 3. Plot of $f^{1 / 2}(E)$ vs. $E$ for the fitting parameters $X$ $=1.78 \mathrm{eV}, Y=0.18 \mathrm{eV}, Z=0.03 \mathrm{eV}$. It is fitted to the straight line equation $0.654(\mathrm{E}-1.68)$.

Malik and O'Leary [7] also reached a similar conclusion albeit for a different reason which is the exponential band tailing at the valence and conduction band edges.

We may also conclude and suggest that may be by joining the two approaches i.e. accounting for exponential band tailing suggested by Malik and O'Leary [7] and our suggestion that the real optical gap does not necessarily start at the valence and conduction bands edges as suggested originally by Tauc (5) might be the right clue to account for the physical origin and the value of the experimental optical energy gap $\left(E_{\text {opt }}\right)$ deduced from the plot due to Cody [4] $\epsilon_{2}^{1 / 2}$ vs. $E$ if it is adopted to analyze $\epsilon_{2}$ data of a-Si-H in the high energy region of the absorption edge.

\section{REFERENCES}

[1] S. K. J. Al-Ani, S. S. Al-Rawi and A. Ibrahim, "The High Region of the Absorption Edge of a-Si:H I, an Analytical Study," World Renewable Energy Congress-IX Florence Italy, 2006.

[2] S. K. J. Al-Ani, A. Ibrahim and S. S. Al-Rawi, "The High Region of the Absorption Edge of a-Si:H II, Derivative Methods Analysis," World Energy Congress-X and Exhibition (WRECX), 2008.

[3] W. B. Jackson, S. M. Kelso, C. C. Tsai, J. W. Allen and S.-J. Oh, "Energy Dependence of the Optical Matrix Element in Hydrogenated Amorphous and Crystalline Silicon," Physical Review B, Vol. 31, No. 8, 1985, pp. 5187-5198. doi:10.1103/PhysRevB.31.5187

[4] G. D. Cody, "The Optical Absorption Edge of a-Si:H," In: J. I. Pankove, Ed., Semiconductors and Semimetals, Academic Press, Waltham, 1984, p. 11.

[5] J. Tauc, R. Grigorovici and A. Vancu, "Optical Properties 
and Electronic Structure of Amorphous Germanium," Physica Status Solidi, Vol. 15, No. 2, 1966, pp. 627-637. doi: $10.1002 / \mathrm{pssb} .19660150224$

[6] A. Ibrahim, "A Theortical Study of the High Absorption Region of the Optical Absorption Edge of Hydrogenated Amorphous Silicon (in Arabic)," Ph.D. Thesis, Baghdad University, Baghdad, 2002.
[7] S. M. Malik and K. O'Leary, "Deviations from SquareRoot Distributions of Electronic States in Hydrogenated Amorphous Silicon and Their Impact upon the Resultant Optical Properties," Journal of Non-Crystalline Solids, Vol. 336, No. 1, 2004, pp. 64-70.

doi:10.1016/j.jnoncrysol.2003.12.042 